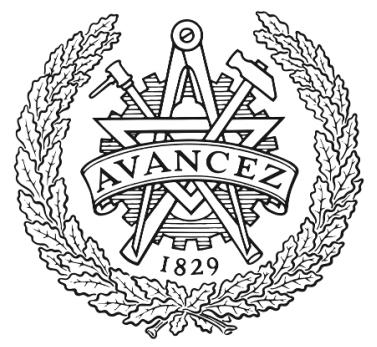

CHALMERS

UNIVERSITY OF TECHNOLOGY

\title{
Temperature-dependent Characterization of Power Amplifiers Using an Efficient Electrothermal Analysis Technique
}

Downloaded from: https://research.chalmers.se, 2023-04-26 13:05 UTC

Citation for the original published paper (version of record):

Taghikhani, P., Buisman, K., Versleijen, m. et al (2022). Temperature-dependent Characterization of Power Amplifiers Using an Efficient Electrothermal

Analysis Technique. IEEE Transactions on Microwave Theory and Techniques, 70(2): 1349-1360. http://dx.doi.org/10.1109/TMTT.2021.3134664

N.B. When citing this work, cite the original published paper.

(O2022 IEEE. Personal use of this material is permitted.

However, permission to reprint/republish this material for advertising or promotional purposes 


\title{
Temperature-dependent Characterization of Power Amplifiers Using an Efficient Electrothermal Analysis Technique
}

\author{
Parastoo Taghikhani, Student Member, IEEE, Koen Buisman, Senior Member, IEEE, Martin Versleijen, \\ Jose-Ramon Perez-Cisneros and Christian Fager, Senior Member, IEEE.
}

\begin{abstract}
In this paper, we propose an efficient methodology for the electrothermal characterization of power amplifier (PA) integrated circuits. The proposed electrothermal analysis method predicts the effect of temperature variations on the key performances of PAs, such as gain and linearity, under realistic dynamic operating conditions. A comprehensive technique for identifying an equivalent compact thermal model, using data from 3-D finite element method thermal simulation and nonlinear curve fitting algorithms, is described. Two efficient methods for electrothermal analysis applying the developed compact thermal model are reported. The validity of the methods is evaluated using commercially available electrothermal computer-aided design (CAD) tools and through extensive pulsed RF signal measurements of a PA device under test. The measurement results confirm the validity of the proposed electrothermal analysis methods. The proposed methods show significantly faster simulation speed comparing to available CAD tools for electrothermal analysis. Moreover, the results reveal the importance of electrothermal characterization in the prediction of the temperature-aware PA dynamic operation.
\end{abstract}

Index Terms-Electrothermal characterization, thermal modeling, thermal network extraction, heat dissipation, thermal impedance identification, power amplifier linearity, EVM.

\section{INTRODUCTION}

$\mathbf{T}$ HE ever increasing demand for faster and more reliable wireless communication imposes rigorous performance requirements on the power amplifiers (PAs) in terms of output power, efficiency, and linearity [1], [2]. In particular, recent time-division duplexing (TDD) systems require power amplifiers with high linearity features, to cope with switching transient effects. In such systems the key metrics for linearity, especially error vector magnitude (EVM), must be very low

P. Taghikhani, Koen Buisman, Jose-Ramon Perez-Cisneros and C. Fager are with the Department of Microtechnology and Nanoscience, Chalmers University of Technology, SE-412 96 Gothenburg, Sweden (e-mails: \{parastoo;k.buisman;jospere;christian.fager\}@chalmers.se). Koen Buisman is also with the Department of Electrical and Electronic Engineering, University of Surrey, Surrey GU2 7XH, United Kingdom (e-mails: k.buisman@surrey.ac.uk). Martin Versleijen is with NXP Semiconductors Company, 6534 AE Nijmegen, Netherlands (e-mail: martin.versleijen@nxp.com).

This work is a part of the Silicon-based Ka-band massive MIMO antenna systems for new telecommunication services (SILIKA) project, funded by the European Union's Horizon 2020 research and innovation program under the Marie Sklodowska Curie grant agreement No \#721732. Part of the work has also been carried out in the GigaHertz Centre in a joint project financed by the Swedish Government Agency for Innovation Systems (VINNOVA), Chalmers, Ericsson, Keysight Technologies, Infineon, Saab, and UMS. The European Union is also acknowledged for the funding and support of the H2020 ICT project SERENA (Grant Agreement no.779305). to meet anticipated high data rate communication [3], [4]. A variety of techniques, such as digital predistortion (DPD), exist for improving EVM [5]-[7]. However, it is particularly challenging to compensate for fast time-varying and transient non-linearity issues [1]-[4], [8], [9].

It is well established that the challenges associated with signal linearity and efficiency are highly affected by the electrothermal transient behavior of PAs. The electrothermal behavior refers to the condition in which the PA electrical operation changes the temperatures of device nodes, and vice versa. Particularly, due to the high level of integration, thermal issues increasingly impact the performance and reliability of high power integrated circuits (ICs). Besides, thermal issues may entail a significant degradation of the performance of the PA ICs, and must be accurately investigated. Therefore, electrothermal analysis of PA ICs is greatly needed at any design stage [10]-[12]. Furthermore, thermal-aware PA designs help in the development of ICs with optimum thermal management.

A common approach for electrothermal analysis is to couple a 3-D thermal solver with an individual electrical solver at each electrical simulation step [13]-[18]. Such a coupled electricalthermal simulation procedure relies on the convergence of the iterative loop between the electrical- and individual thermal solvers. Consequently, the simulation procedure is timeconsuming and computationally expensive, although accurate temperature-aware results can be obtained. On the other hand, it is well known that the heat diffusion problem can be numerically discretized using the finite element method (FEM) and represented as an equivalent thermal circuit [19], [20]. The thermal equivalent circuit can be integrated into the electrical simulation framework for an efficient electrothermal analysis. The size of the discretized heat problem can be considerably reduced through model order reduction (MOR) techniques [21]-[28]. However, the computational cost for the electrothermal analysis of complex electronic circuits remains high even if MOR approaches are used.

The necessity to reduce computational complexity leads to the development of the Compact Thermal Model (CTM) for complex circuits [10], [20], [29]-[33]. The compact thermal network can be obtained from post-processing 3D-FEM thermal simulation data when only the behavior of specific nodes is considered for thermal modeling. The aforementioned thermal modeling approach creates possibilities in which a desired trade-off between accuracy and complexity can be achieved [10], [29]. 
In this paper, first, an efficient algorithm for compact thermal model extraction of the PA ICs is proposed. The algorithm develops a thermal model using the step response of the thermal system while specific areas, e.g. transistors, are treated as heat sources or interface nodes. The dynamic selfheating and thermal coupling behavior of interface nodes are obtained and fitted to suitable mathematical functions in timeand frequency domains.

The thermal model is then used for electrothermal analysis to predict temperature-aware PA performance. In this paper, two efficient methods for electrothermal analysis are developed. A circuit-based electrothermal analysis method is presented where the thermal equivalent circuit is incorporated into an electrical solver (e.g. Keysight ADS or Spice). The circuitbased method can be used to perform accurate thermallyaware prediction in any type of circuit simulation. The second approach is a model-based electrothermal analysis method, which combines PA nonlinear behavioral model with the PA thermal model in an iterative algorithm, suitable for envelope time-domain predictions. The latter approach is less timeconsuming for investigating PA dynamic operation under complex modulated signal excitation compared to using circuitbased electrothermal simulation. The proposed methods are generic and developed independently of the PA characteristics.

The thermal modeling and both electrothermal analysis approaches are verified through extensive pulsed-RF measurements and predictions with the commercial Keysight ADS Electro-Thermal Simulator. The proposed electrothermal approaches show considerably shorter simulation times compared to the commercial simulator. Furthermore, the linearity results reveal the importance of electrothermal analysis for accurate prediction of the dynamic operation mode of PAs under realistic, pulsed modulated signal excitations present in modern communication systems. Therefore, the proposed electrothermal analysis method can be exploited at the PA design stage to develop proper compensations for the problems of heat dissipation.

This paper is organized as follows: Section. II presents an overview of the thermal modeling and the corresponding identification algorithm. In Section. III the thermal modeling technique is applied to a PA device under test (DUT) and the corresponding thermal modeling results are presented. Section. IV describes the two proposed approaches for efficient electrothermal simulation. The validity of the circuit-based electrothermal analysis is assessed in this section. Section. V presents the experimental setup and results for the electrothermal characterization of the PA DUT. In this section, the PA performance under static and pulsed-signal excitation is evaluated using the proposed model-based simulation technique. Finally, Section. VI gives concluding remarks.

\section{Thermal MOdel EXTRACTION}

It is well-known that, once discretized, the heat wave equation in a homogeneous and linear domain, can be represented by a reciprocal and passive thermal network model [10], [19], [29]. In this section, following the compact thermal modeling approach, the concept of the thermal impedance matrix and the corresponding model identification are described.

\section{A. Transient thermal impedance matrix}

Let us consider a device including $M$ designated areas as heat sources. The elements of the thermal impedance matrix, $\mathbf{Z}_{t h}(t)$, assuming a linear thermal system, are defined by [10]:

$$
\begin{gathered}
z_{t h, i j}(t)=\frac{\Delta T_{i}(t)}{P_{j}}, \\
\Delta T_{i}(t)=T_{i}(t)-T_{\mathrm{amb}}
\end{gathered}
$$

where $T_{i}(t)$ is the temperature of the $i^{\text {th }}$ heat source at time instant $t$ when port $j$ is excited with a step power i.e. $P_{j}$. $T_{\text {amb }}$ is the ambient temperature. $z_{t h, i i}$ represents the selfheating thermal impedance and $z_{t h, i j}, i \neq j$ is the mutual thermal impedance, indicating thermal coupling between ports. The steady-state value of thermal impedance, i.e. $t \rightarrow \infty$, corresponds to the thermal resistance.

Once the desired terminals corresponding to heat sources areas are specified, the system step response can be obtained using a suitable 3D-FEM thermal simulator. For active circuits, areas with high power dissipations such as transistors fingers and resistors can be specified as heat sources. The elements of thermal impedance matrix can be represented in time- or frequency domain. Particularly, it is shown that the transfer function of a linear thermal problem can be synthesized with an equivalent Foster network [21], [30]. Therefore, the elements of the multi-port thermal impedance can be written in time domain as

$$
z_{t h, i j}(t)=\sum_{n=1}^{N_{m}} c_{n, i j}\left(1-e^{-\frac{t}{\tau_{n}}}\right)
$$

where $N_{m}$ is the number of time constants, i.e $\tau_{n}$. Besides, $c_{n, i j} \geq 0$ and $\tau_{n}>0$ to ensure a passive positive solution to the thermal diffusion problem [10], [34].

\section{B. Identification algorithm}

As it has been described, transient temperature data is required to obtain the thermal impedance matrix. For this purpose, each heat source needs to be excited with a step power and the transient temperature on all terminals must be collected. In this way, each rows of $\widetilde{\mathbf{Z}}$ matrix of temperature data, representing the system step response, is created. The $\widetilde{\mathbf{Z}}$ is then used to find the thermal impedance model, as introduced in (3).

Fig. 1 shows the flowchart of the iterative algorithm for identifying the coefficients of the thermal model. For a device with $M$ specified heat sources, the thermal impedance matrix, $\mathbf{Z}_{t h}$, has a size of $M \times M$. Therefore, from the thermal simulation, $M^{2}$ temperature step responses, including selfheating and thermal mutual coupling, exist. The thermal system is assumed to be linear and time-invariant. Therefore, time constants, i.e. $\tau_{n}$, are common for all elements of thermal impedance matrix. In addition, the model linear coefficients, $c_{n, i j}$, are unique for each element of the thermal impedance matrix. Details of each step in the identification algorithm are presented below.

Step 1 (Initial guess): First, the algorithm starts with assigning an initial guess for the time constants. i.e. $\tau_{n}^{(0)}, n=$ $1, \ldots, N_{m}$. Thus, $\mathbf{Z}_{t h}\left(t, \tau_{n}^{(0)}\right)$ can be evaluated. 


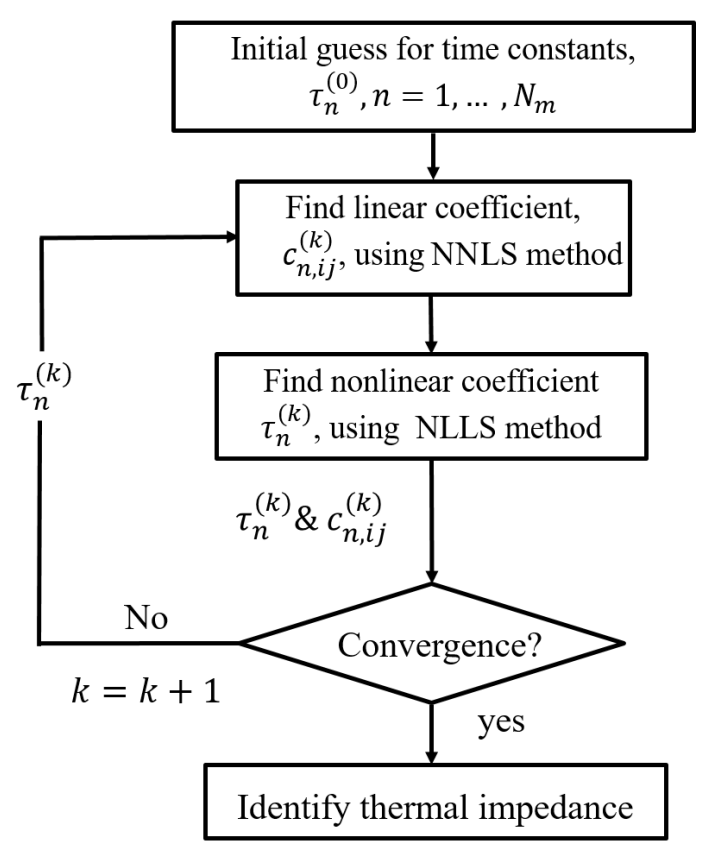

Fig. 1. The identification algorithm based on linear and nonlinear least square method.

Step 2 (Find linear coefficients): For the $k^{\text {th }}$ iteration, the linear coefficients, $c_{n, i j}^{(k)}$, are found by non-negative linear least square (NNLS) estimator considering that $c_{n, i j}^{(k)} \geq 0$ as follows

$$
c_{n, i j}^{(k)}=\operatorname{argmin}\left\|\mathbf{Z}_{t h}\left(t, \tau_{n}^{(k-1)}\right)-\widetilde{\mathbf{Z}}\right\|
$$

where $\|$.$\| is the 2-norm matrix operator. Due to non-negativity$ constraints, a pseudoinverse solution is not sufficient to find linear coefficients. However, there exists several fast and efficient algorithms to solve a NNLS problem. In particular, MATLAB provides a built-in function to solve NNLS problems.

Step 3 (Find time constants): Once $c_{n, i j}^{(k)}$ are identified, the new time constants, which corresponds to the nonlinear model coefficient, can be found using nonlinear least square algorithm.

$$
\tau_{n}^{(k)}=\operatorname{argmin}\left\|\mathbf{Z}_{t h}\left(t, c_{n, i j}^{(k)}\right)-\widetilde{\mathbf{Z}}\right\|
$$

It should be noted that the nonlinear least square problem can be solved by using standard curve fitting algorithms.

Step 4 (Check convergence): The algorithm ends when the residue or the fitting error, $\epsilon=\left\|\mathbf{Z}_{t h}\left(t, \tau_{n}^{(k)}, c_{n, i j}^{(k)}\right)-\widetilde{\mathbf{Z}}\right\|$, has stabilized to a minimum value.

For the thermal impedance matrix identification, $M^{2}$ curves are mapped simultaneously to the analytical function introduced in (3).

\section{PA Thermal Model}

Thermal model identification is essential for the electrothermal characterization of PAs. In this section, the algorithm proposed in Section. II-B for the thermal model identification has been evaluated through modeling of a PA DUT.

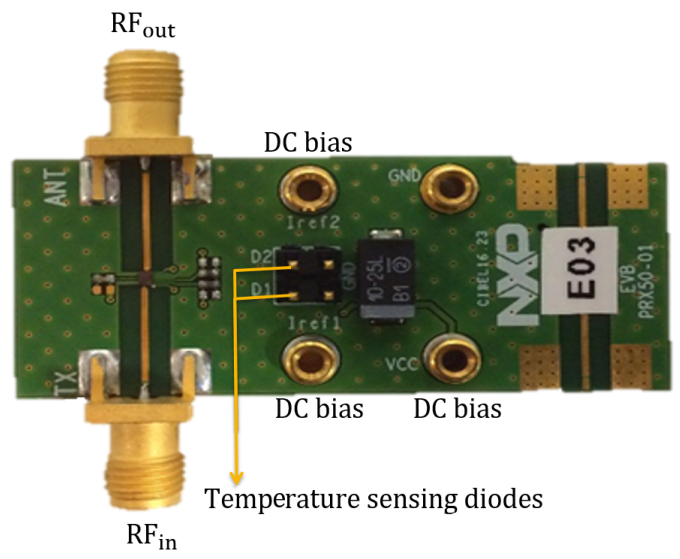

Fig. 2. Photo of the PA DUT evaluation board including temperature sensing diodes.

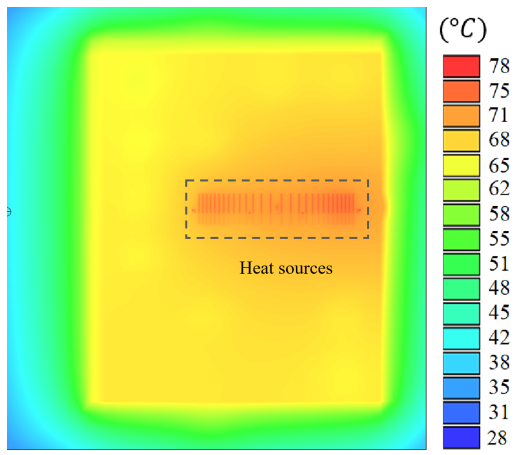

Fig. 3. Location and steady state temperature of heat sources on die obtained from ADS HeatWave 3D thermal simulator.

\section{A. PA DUT}

A two-stage class $\mathrm{AB}$ BiCMOS amplifier manufactured by NXP Semiconductor has been selected to test the proposed algorithm for thermal model identification. It operates at 5$6 \mathrm{GHz}$ and is equipped with temperature-sensing diodes for electrothermal characterization and monitoring. The PA DUT is used for investigating linearity challenges associated with wireless communications. Additionally, the PA includes integrated sensing diodes for temperature measurements. Fig. 2 shows a photo of the PA evaluation board.

\section{B. Thermal modeling results}

Once the location of the heat sources and the thermal properties of the layout are specified, a 3D-FEM thermal simulator can be used for its thermal characterization. The critical power dissipating areas such as transistors and resistors are examples of heat sources which are relevant from an electrical viewpoint. It is recommended to specify high-power transistors fingers as heat sources to obtain an accurate thermal model. However, the thermal model would be more accurate if more areas are defined as heat sources and there is always a trade-off between accuracy and modeling efficiency which should be considered. As described in Section. II-B, thermal simulation data includes the temperature rise of the heat sources when they are excited by a step power. 
TABLE I

IDENTIFIED THERMAL TIME CONSTANTS FOR THE POWER AMPLIFIER DEVICE UNDER TEST.

\begin{tabular}{cccccccccccccc}
\hline$\tau_{1}$ & $\tau_{2}$ & $\tau_{3}$ & $\tau_{4}$ & $\tau_{5}$ & $\tau_{6}$ & $\tau_{7}$ & $\tau_{8}$ & $\tau_{9}$ & $\tau_{10}$ & $\tau_{11}$ & $\tau_{12}$ & $\tau_{13}$ & $\tau_{14}$ \\
\hline $1.75 \mathrm{e}-08$ & $3.55 \mathrm{e}-06$ & $1.65 \mathrm{e}-07$ & $9.07 \mathrm{e}-07$ & $9.00 \mathrm{e}-06$ & $7.12 \mathrm{e}-05$ & $2.33 \mathrm{e}-04$ & $6.08 \mathrm{e}-04$ & $2.37 \mathrm{e}-05$ & $1.73 \mathrm{e}-02$ & $1.99 \mathrm{e}-02$ & $1.64 \mathrm{e}-03$ & $1.45 \mathrm{e}-01$ & $1.04 \mathrm{e}+01$ \\
\hline
\end{tabular}

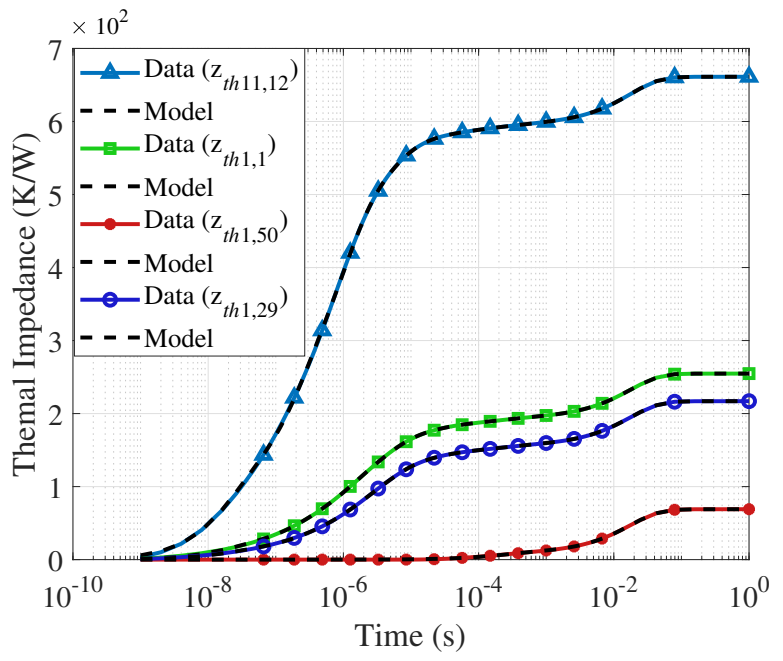

Fig. 4. Thermal impedance identification of the PA DUT for some elements. Each heat source is excited with $20 \mathrm{~mW}$ step input power.

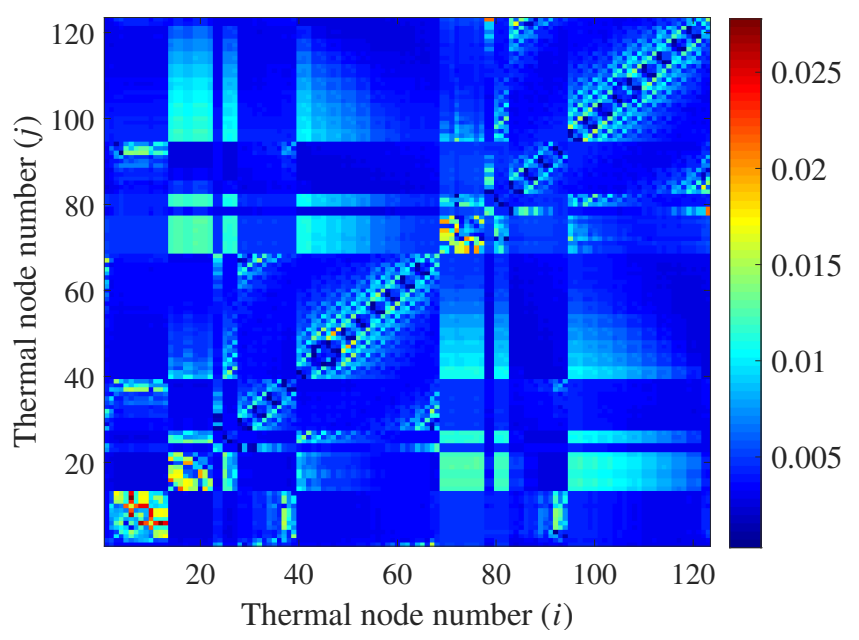

Fig. 5. RMS error of identification algorithm for all the elements of $123 \times 123$ thermal impedance matrix.

In total, $M=123$ individual areas, corresponding to diodes and transistors fingers, were specified as heat sources in the PA DUT layout. Thermal simulations were performed using the ADS Heatwave 3-D thermal simulator. The initial guess for the 14 values of time constants is to have them logarithmically spaced over the considered time interval. HeatWave runs in a standalone mode as a pure thermal solver and was decoupled from the ADS electrical solver for the studies presented here. The thermal technology file, including material thermal properties from the process design kit (PDK), was provided as input to the ADS HeatWave simulator. Fig. 3 shows an example of HeatWave thermal simulation results, representing steady-state temperature on the device given nominal DC dissipated power. However, for identifying the thermal model, each heat source was excited separately with a $20 \mathrm{~mW}$ step input power, and the temperature rise versus time of all terminals was collected. It took approximately 2 hours to extract $123 \times 123$ temperature step responses, i.e. $\widetilde{\mathbf{Z}}$, using logarithmic time step setting.

The model identification algorithm described in Section II-B has converged after 25 iterations and took about 20 minutes to run on a Core i7 desktop PC with $32 \mathrm{~GB}$ of RAM. The thermal model was identified with 14 time constants, ranging between $10 \mathrm{~ns}$ and $10 \mathrm{~s}$, see Table I. Fig. 4 gives the modeled and 3-D thermal simulation results for four of the thermal impedances, i.e. $z_{t h 11,12}, z_{t h 1,1}, z_{t h 1,50}$ and $z_{t h 1,29}$. The modeled thermal impedance shows accurate results for all the matrix elements. The root mean square (RMS) error of the model fitting is below $3 \%$ for every element of the impedance matrix, see Fig. 5. The developed thermal model has been used for the electrothermal analysis of the PA DUT. In the following section, two approaches for electrothermal analysis are described based on the developed thermal model. As is mentioned in Section II, the model is extracted based on the thermal linearity assumption. Therefore, if the device temperature goes into a nonlinear thermal region, the thermal model becomes inaccurate. In such cases, the temperature dependency of the material must be included in the model extraction.

\section{ELECTROTHERMAL SIMULATION APPROACHES}

Electrothermal analysis refers to a temperature-aware electrical simulation. Coupled electro-thermal simulation using dedicated computer aided design (CAD) tools is one way to perform electrothermal analysis. In this approach, a circuit simulator computes power dissipation and a thermal simulator computes device temperatures. Temperatures and power dissipation are exchanged between the two simulators through interface nodes until a self-consistent solution is achieved [13], [15], [17]. Usually, interface nodes are the device nodes corresponding to temperature-dependent active circuit elements. Therefore, temperature-aware circuit performance can be achieved accurately. However, such an approach is time-consuming and computationally expensive. On the other hand, once the thermal behavior of the IC is properly modeled, it can be used instead of running 3-D FEM thermal simulation in an electrothermal analysis procedure. The use of the thermal model, or the equivalent thermal circuit, can improve the speed and capacity of electrothermal analysis considerably.

In this paper, based on a thermal modeling approach, two electrothermal analysis methods are proposed for the efficient 


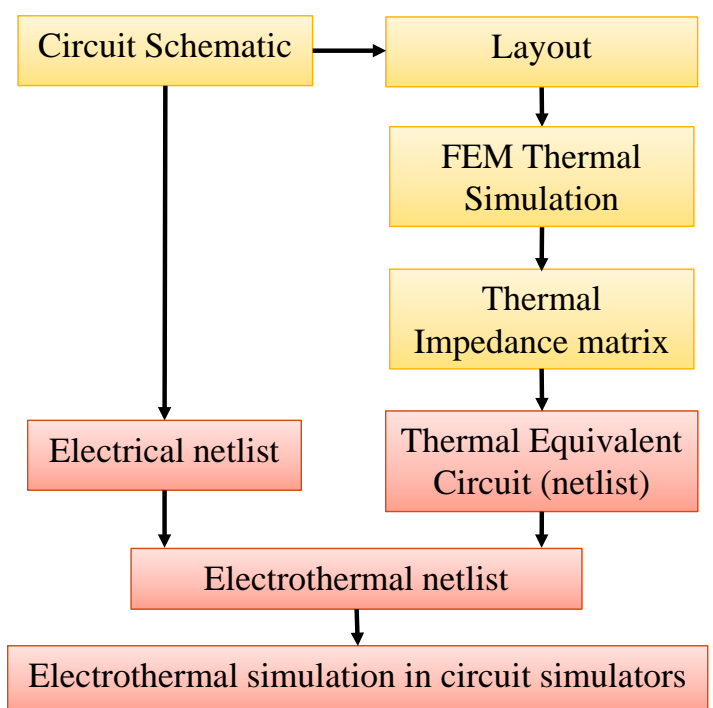

(a)

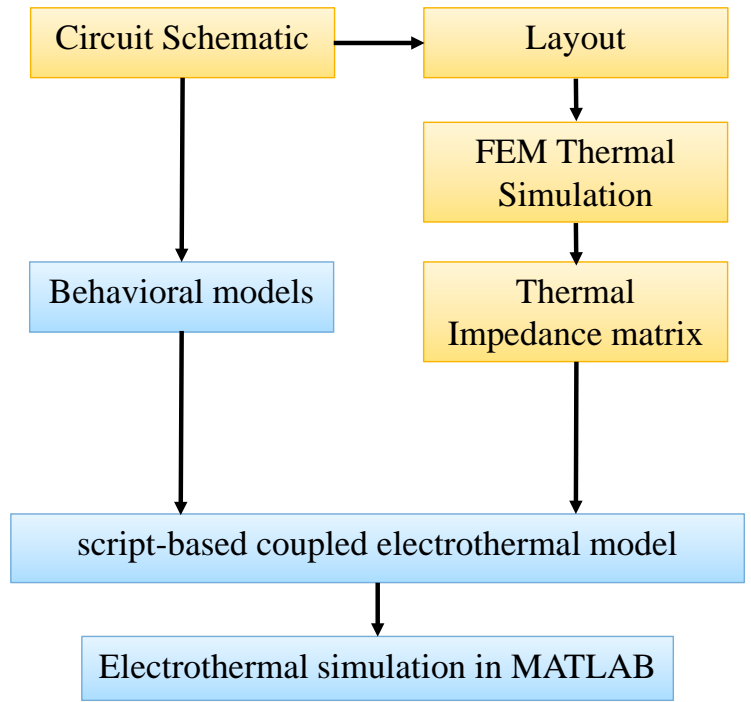

(b)

Fig. 6. The electrothermal analysis approaches: a) circuit-based simulation, and b) model-based simulation.

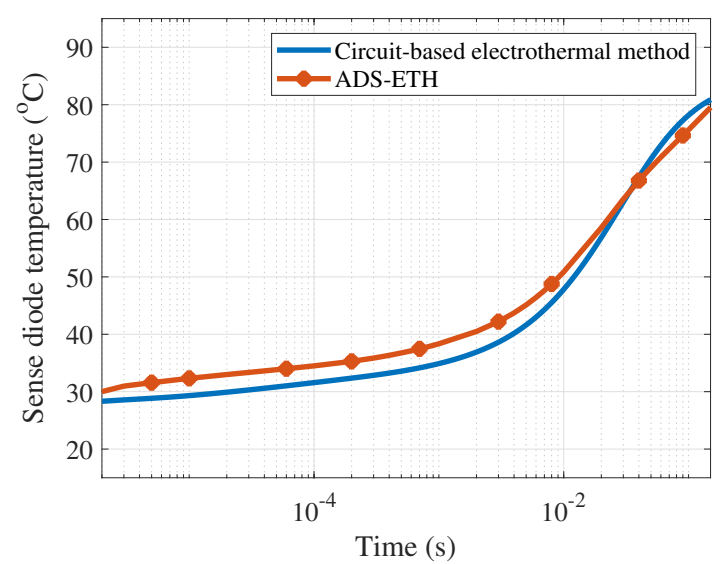

Fig. 7. Sense diode temperature obtained from ADS-ETH and the circuitbased electrothermal method when transient simulation was performed. The PA DUT is not RF excited and DC biases are step current and voltage sources with $2 \mu$ s rise time and $2 \mu$ s delay.

temperature-aware simulation of PAs. Fig. 6(a) illustrates the circuit-based electrothermal analysis, in which an equivalent thermal circuit is incorporated into an electrical simulation for coupled electrothermal analysis. The model-based approach, on the other hand, relies on PA nonlinear behavioral modeling coupled with evaluation of thermal impedance matrix in an iterative algorithm, see Fig. 6(b).

\section{A. Circuit-based electrothermal simulation}

It is crucial to provide a thermal equivalent circuit topology that is compatible with common circuit simulators. By definition, the elements of the thermal impedance matrix, $z_{t h, i j}$, represents system step response. To extract an equivalent thermal circuit the impulse response, $z_{i m p, i j}$, is needed [10].
For the proposed thermal impedance model in Section. II, the frequency domain representation of $z_{i m p, i j}$ is given by

$$
z_{i m p, i j}(s)=\sum_{n=1}^{N_{m}} \frac{c_{n, i j}}{1+\tau_{n} s}
$$

The equivalent circuit development is a well-established area in thermal modeling field [10], [19], [21], [23], [30], [35]. In this paper, a circuit topology similar to [35], including Voltage Control Current Source (VCCS) and Current Control Current Source (CCCS) elements is developed. The VCCSs in this circuit are compatible with the HSpice Foster pole-residue format, and their values correspond to $z_{i m p, i j}$ in frequency domain obtained from (6) [36]. An extra node including a $1 \mathrm{~K} / \mathrm{W}$ resistor and a CCCS also exist per thermal node to generate the required controlled voltages for VCCSs.

The proposed topology creates $2 \times M$ nodes that will be added to the electric circuit netlist. The number of nodes does not depend on the number of time constants and is proportional to the number of heat sources. The proposed topology and the use of VCCSs in Foster pole-zero format makes the thermal equivalent circuit highly efficient for electrothermal analysis.

The thermal netlist has been integrated as a sub-circuit into the PA electrical netlist. Temperature-dependent active circuit elements were updated with an additional thermal node representing the temperature. The effect of self- and mutual heating were accurately taken into account using the thermal equivalent circuit obtained from the multi-port thermal impedance matrix. The PA temperature-aware performance has been assessed using the proposed circuit-based electrothermal analysis method.

\section{B. Circuit-based electrothermal simulation results}

A variety of electrothermal simulations have been performed for evaluating the proposed circuit-based electrothermal 


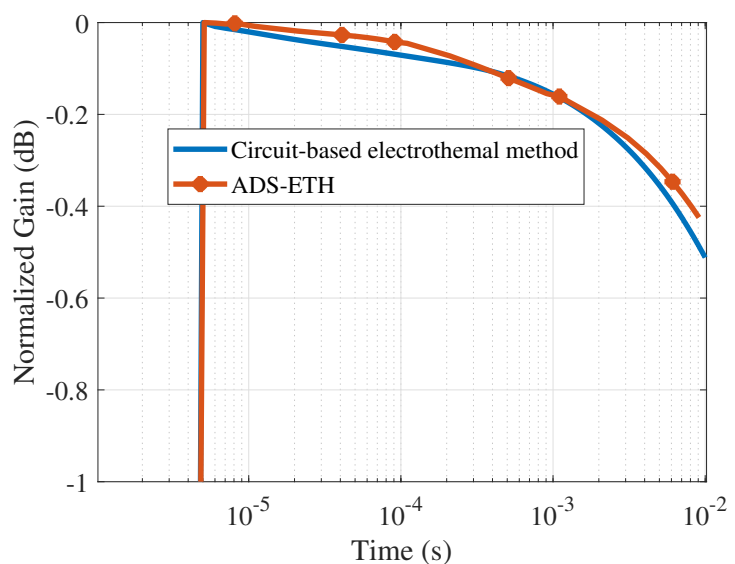

(a)

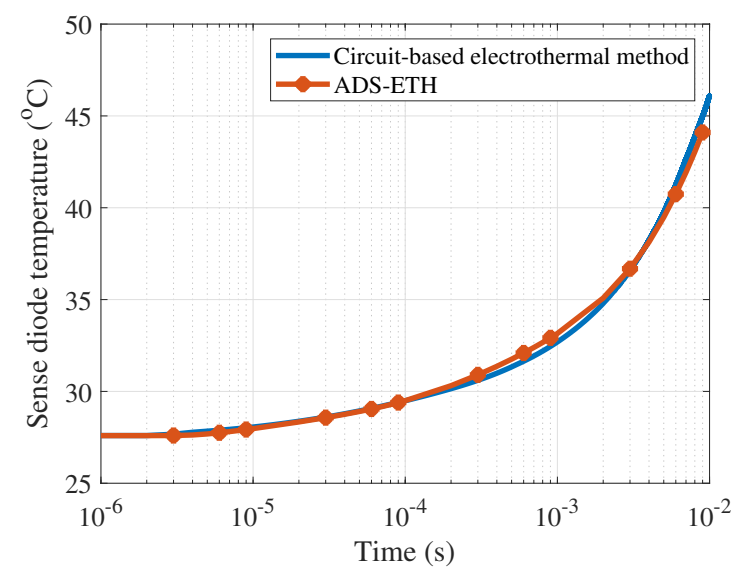

(b)

Fig. 8. Envelope simulation results obtained from ADS-ETH and the proposed circuit-based electrothermal method. The PA DUT input is an RF step power source with $-20 \mathrm{dBm}$ average power and $2 \mu$ s rise time and $2 \mu$ s delay. DC biases are also pulsed DC voltages and current sources with $2 \mu$ s rise time and $2 \mu$ s delay: a) Gain variation versus time, b) sense diode temperature versus time.

analysis methodology. The simulation results have been verified by the Keysight ADS Electro-Thermal Simulator, which is here called ADS-ETH for simplicity [17]. The ADS-ETH solver iterates a full 3D thermal solver, i.e. HeatWave, with the ADS circuit simulator in each simulation point until a converged solution is achieved. In this way, accurate electrothermal analysis of the PA can be obtained although the simulation speed is limited.

The PA DUT DC bias was provided by two $600 \mu \mathrm{A}$ base current sources and a $4.8 \mathrm{~V}$ collector voltage source in the simulation schematic. The temperature sensing diodes were also biased with $10 \mu \mathrm{A}$ constant current sources and showed almost identical temperature behaviors. Fig. 7 shows the simulated temperature variation of one of the sensing diodes versus time when the DC biases were switched on at $t=2 \mu$ s using step sources with $2 \mu$ s rise-time. The sensing diode temperature reaches almost the steady-state value after $100 \mathrm{~ms}$ when the PA has no RF input excitation, corresponding to the self heating that the DC biasing causes. The proposed circuit-based electrothermal analysis results agree well with
TABLE II

EVALUATION TIME PER SIMULATION POINT (SECONDS)

\begin{tabular}{lcc}
\hline ADS solver & ADS-ETH & Circuit-based electrothermal \\
\hline Transient simulation & 122 & $1 \mathrm{e}-3$ \\
Envelope simulation & 107 & 0.2 \\
\hline
\end{tabular}

ADS-ETH results. As it can be seen in Fig. 7, the temperature results difference between the two methods is less than 2 degrees.

Fig. 8 presents the envelope simulation results in which a $5.9 \mathrm{GHz}$ continuous wave $(\mathrm{CW})$ step input power with $2 \mu \mathrm{s}$ rise-time, $2 \mu$ s delay, and $-20 \mathrm{dBm}$ power was the PA input excitation. The DC biases were also switched on at $t=2 \mu \mathrm{s}$. Therefore, the temperature began to rise from the ambient temperature value. Fig. 8 (a) shows temperature-aware envelop simulation results in which PA gain is reduced by about $0.4 \mathrm{~dB}$ after $10 \mathrm{~ms}$. PA thermal nodes and sensing diode temperatures were also computed in this simulation and shown in Fig. 8 (b). The proposed circuit-based electrothermal simulation results agreed very well with the ADS-ETH results.

The extensive performed simulations have verified the accuracy of the proposed circuit-based electrothermal method. Furthermore, the proposed electrothermal method shows a few orders of magnitude faster simulation speed compared to ADS-ETH, see Table. II. Clearly, using an equivalent thermal network can improve the efficiency and speed of electrothermal simulation, although, it comes at the price of an additional thermal model identification step. It should be noted that the procedure of the thermal network extraction can be added to circuit simulators such as ADS or Spice in a fully automated way.

\section{Model-based electrothermal simulation}

The circuit-based electrothermal analysis presented above shows relatively faster performance compared to ADS-ETH. However, for investigating PA performance under modulated signal excitation, the circuit-based electrothermal simulation time is still too long. In this paper, an alternative approach is therefore proposed, in which PA nonlinear behavioral modeling techniques is employed for the electrothermal analysis. A similar approach was used for multi-branch transmitters in [37]-[39], but is here modified for electrothermal analysis of a single PA having a multi-port thermal impedance model.

Fig. 9 shows an illustration of the joint simulation algorithm for the model-based electrothermal analysis. The inputs to begin the algorithm are the initial temperature and input signal values. In each time instant, the PA dissipated power and RF output signal are calculated using PA nonlinear behavioral models. For the next time instant, the temperature is obtained from the PA thermal model and the modeled dissipated power. The new temperature value is considered as the PA temperature in the next time instant and used to find the PA output from the nonlinear behavioral models. The algorithm runs through all time steps of the input signal sequence. The iterative algorithm 


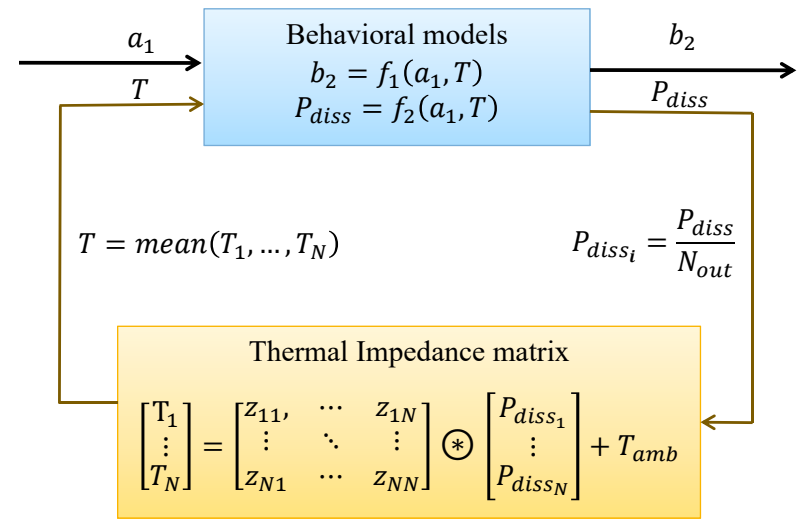

Fig. 9. The model-based electrothermal analysis approach. $N_{\text {out }}$ is the number of PA output transistors and $\circledast$ is the convolution symbol.

is explained extensively in [37]-[39]. It should be noted that these simulations run in the complex envelope/modulation time domain.

1) PA nonlinear behavioral model: The PA output and dissipated power depend on the PA input signal, and temperature. Therefore, the temperature-dependent model is obtained by repeating the PA nonlinear model extraction at different ambient temperatures. Typically, the PA thermal response is much slower than the PA response to the RF signal. Therefore, the nonlinear model for PA output and dissipated power, following a static polynomial modeling approach, can be written as [38], [39]:

$$
\begin{gathered}
b_{2}\left(a_{1}, T\right)=\sum_{p_{1}=1}^{P_{1}} \alpha_{p_{1}}(T) a_{1}\left|a_{1}\right|^{2\left(p_{1}-1\right)} \\
P_{\text {diss }}\left(\left|a_{1}\right|, T\right)=P_{d c}+P_{\text {in }}-P_{\text {out }}=\sum_{p_{d}=0}^{P_{d}} \xi_{p_{d}}(T)\left|a_{1}\right|^{p_{d}}
\end{gathered}
$$

where $a_{1}$ and $b_{2}$ are complex baseband/envelope representations of the PA input and output, respectively. $T$ is temperature and $\alpha_{p_{1}}(T)$ and $\xi_{p_{d}}(T)$ are temperature-dependent model coefficients.

Fig. 10 presents the temperature-dependent PA behavior in terms of amplitude to amplitude (AM/AM), amplitude to phase (AM/PM), and dissipated power. The results were obtained from performing circuit simulations of the PA DUT when the thermal effects, i.e. thermal network, were disabled. Such data can also be obtained from pulsed RF measurements at different ambient temperatures. With $P_{1}=9$ and $P_{d}=6$ model coefficients in (7) and (8), there is a good agreement between the modeled and simulated results.

2) PA simplified thermal model: The formulation of the multi-port thermal impedance is introduced in Section II. The thermal impedance matrix provides the temperature of individual heat sources taking into consideration self-heating and thermal coupling. The PA power consumption is mainly dissipated in the output transistors. Therefore one can assume that total dissipated power is equally distributed between heat sources belonging to the PA output transistors. The

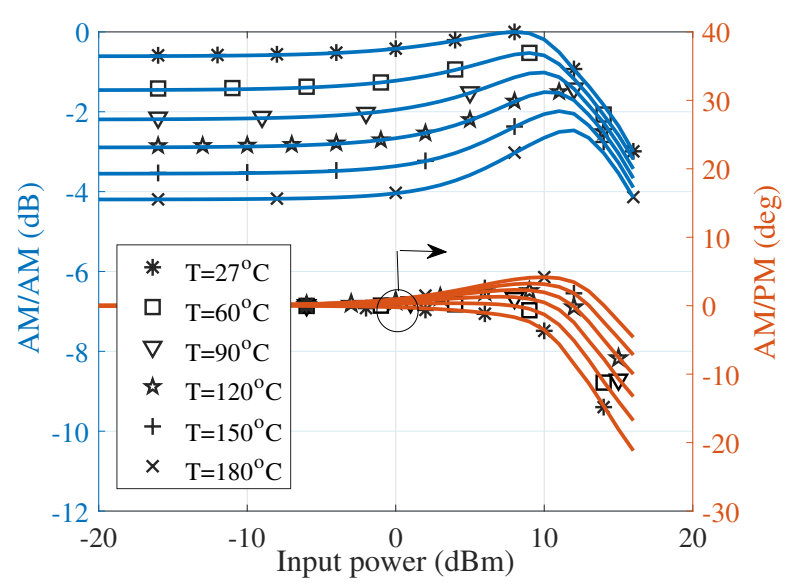

(a)

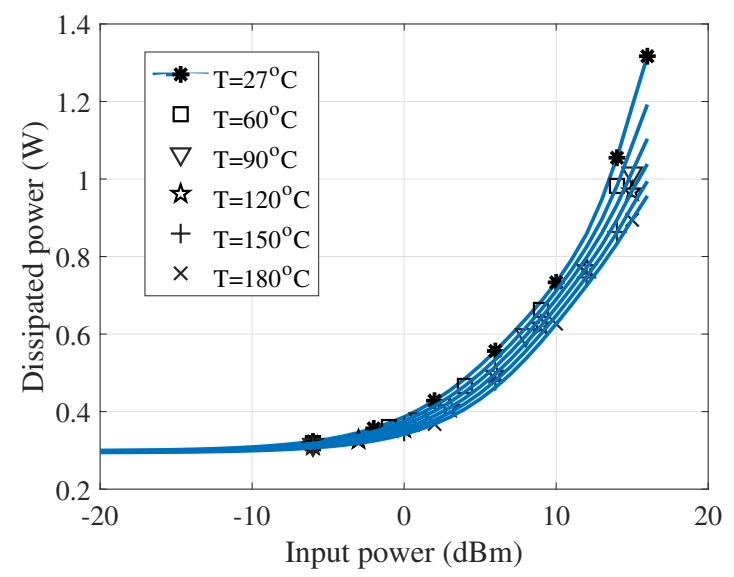

(b)

Fig. 10. Modeled and simulated power amplifier (PA) characteristics at six different temperatures: (a) gain variation / $\mathrm{AM} / \mathrm{AM}$ and phase variation / AM/PM, (b) dissipated power (Pdiss). Markers and solid lines represent simulated and modeled results, respectively. AM/AM graphs are normalized to the maximum value of $\mathrm{PA}$ gain related to $27^{\circ} \mathrm{C}$ ambient temperature.

overall PA temperature can be approximated as the average of temperatures corresponding to the output transistors heat sources. In total 123 areas including diodes and transistors are specified as heat sources for the PA DUT in Section III. The number of output transistors, $N_{\text {out }}$, is 84 . Therefore, the dissipated power of each heat source is approximated by

$$
P_{\text {diss }_{i}}(t)= \begin{cases}\frac{P_{\text {diss }}(t)}{N_{\text {out }}}, & i=1, \ldots 84, \in \text { PA output transistors. } \\ 0, & \text { otherwise. }\end{cases}
$$

where $P_{\text {diss }}(t)$ is the total instantaneous power dissipated in the PA. Assuming the temperature of output transistors behave similarly, the PA's approximated temperature can be written as

$$
\begin{gathered}
T(t) \approx \operatorname{mean}\left(T_{1}(t), \ldots, T_{N_{\text {out }}}(t)\right) \approx T_{i}(t) \\
T_{i}(t)=\sum_{j=1}^{N_{\text {out }}} z_{i m p, i j}(t) \circledast P_{d i s s_{j}}(t)
\end{gathered}
$$




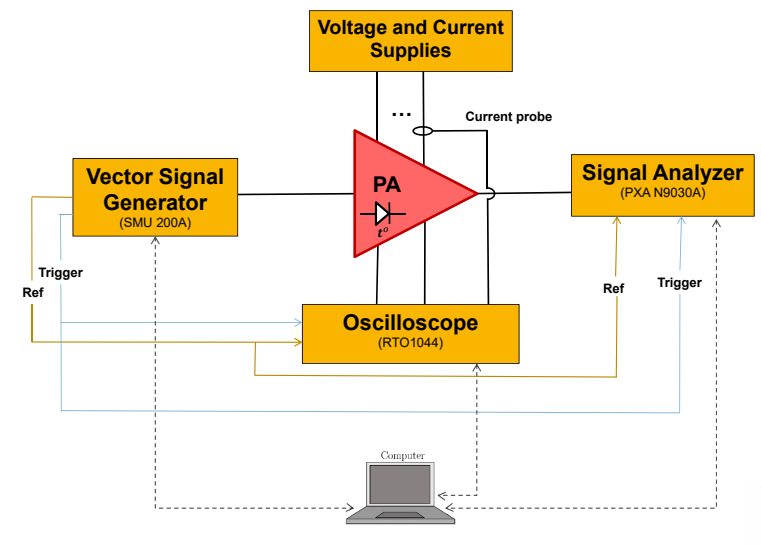

Fig. 11. Block diagram of pulsed RF measurement setup for evaluation of proposed electrothermal method. The PA is equipped with sensing diodes. The reference and trigger signal are required for correct pulse to pulse synchronization

where $\circledast$ is the convolution operator

$$
z_{i m p, i j}(t) \circledast P_{d i s s_{j}}(t)=\int_{0}^{t} z_{i m p, i j}(t-\tau) P_{\text {diss }_{j}}(\tau) d \tau
$$

$z_{i m p, i j}(t)$ are the elements of the PA impulse response matrix in the time domain, which can be simply obtained from (6). One can solve (11) by implementing discrete convolution.

These approximations have been considered for simplifying the PA thermal model in the model-based electrothermal analysis implementation. Once the PA thermal and nonlinear behavioral models are extracted, the simulation algorithm from Fig. 9 can be implemented in MATLAB or any other numerical computing software. Therefore, the electrothermal analysis can be performed independent from a circuit simulator.

The PA performance under pulsed RF and modulated signal has been evaluated using the model-based electrothermal analysis and corresponding measurements. In the following section, the pulse measurement setup and results are reviewed.

\section{EXPERIMENTAL VERIFICATION}

Measurements with various pulsed RF signal excitations were performed to verify the proposed model-based electrothermal analysis approach. A detailed assessment of the proposed method is performed by evaluating the measured and predicted dynamic behavior of the PA response (gain, linearity, etc.). In these experiments, the PA kept heating and cooling during the turning on and off of the PA, respectively. Moreover, the PA temperature variations were measured using one of the embedded sensing diodes and compared with simulation results, as explained in Section IV-B.

\section{A. Experimental Setup}

Fig. 11 shows the block diagram of the experimental setup used for the pulsed characterization. A vector signal generator (Rohde \& Schwarz SMU 200A) was used for generating pulsed signals, and a signal analyzer (Keysight PXA N9030A) for capturing the PA output signal. Constant current supplies (Keithley 2400) were used for biasing the PA base and diodes

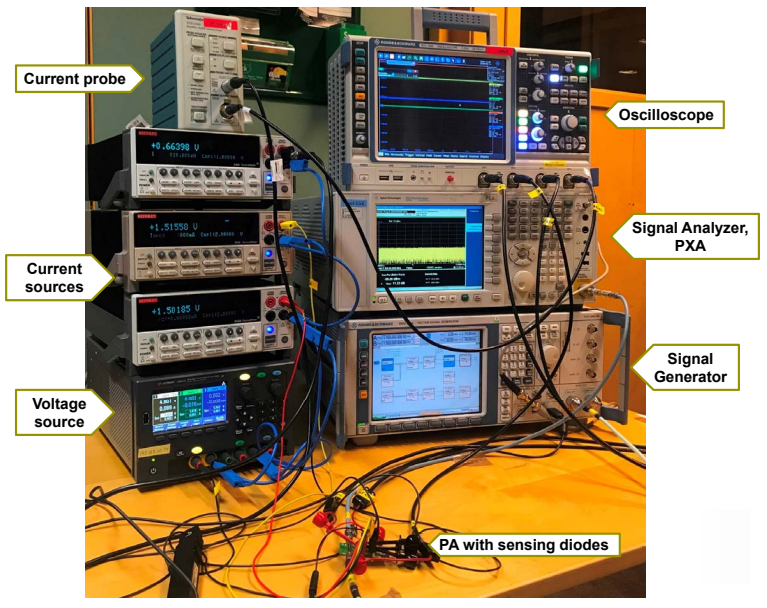

Fig. 12. Measurement setup used for evaluating the PA performance and measuring PA temperature using the embedded sensing diodes.

with a constant current of $600 \mu \mathrm{A}$ and $10 \mu \mathrm{A}$, respectively. The PA collector was biased with a $4.8 \mathrm{~V}$ voltage supply. An oscilloscope (Rohde \& Schwarz RTO 1044) was used to capture one of the sensing diodes voltage. A current probe (Tektronix TCPA 300) was used to measure dynamic DC current. Fig. 12 shows the picture of the measurement setup. The measurement instruments were synchronized via external triggering and a $10 \mathrm{MHz}$ reference signal, which was provided from the signal generator.

\section{B. Static characterization}

Fig. 13 shows the measured static AM/AM compared with the circuit- and behavioral modeling based simulation results. The electrothermal circuit-based simulation has been performed in ADS with the developed electrothermal netlist, as described in Section IV-A. The model-based results were obtained using the algorithm and models presented in Section IV-B. The simulated dissipated power in Fig. 10 was adjusted by a factor of 1.3 to agree with measured DC dissipated power. In order to emulate realistic PA DC biasing conditions, the algorithm runs for one second with $a_{1}=0$ to reach steadystate operating temperature. Then, for each level of input power, the PA output $b_{2}$ was calculated to extract temperatureaware AM/AM characteristics. Moreover, the algorithms runs up to $t=100 \mathrm{~ms}$ for each level of input power to ensure that a stable PA output is reached. Fig. 13 shows that there is a good agreement between measurement and temperature-aware simulation results obtained from both of the proposed methods for PA static operation in terms of gain and efficiency.

\section{Pulsed RF characterization}

Thermal issues affect the PA performance in terms of static and dynamic gain degradation. The dynamic gain variation, i.e. gain variation versus time, can carefully be measured with the pulsed RF measurements. Therefore, two pulsed RF signals with different input powers and pulse-widths have been applied to the PA DUT.

Fig. 14(a) shows the PA gain versus time for input pulses having $20 \%$ duty cycle and $20 \mathrm{~ms}$ and $40 \mathrm{~ms}$ pulse-widths, 


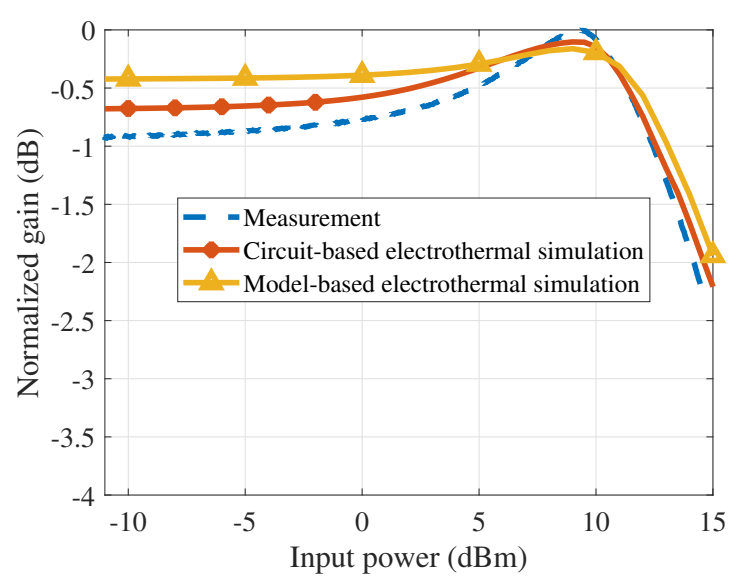

(a)

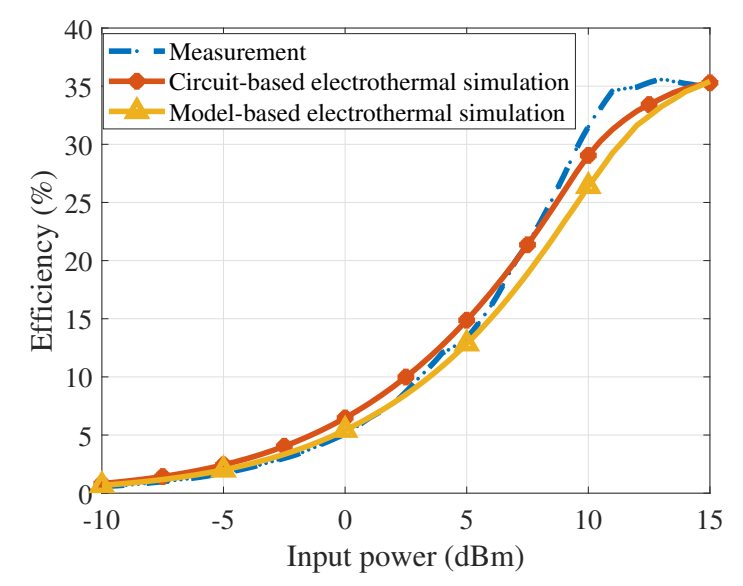

(b)

Fig. 13. The PA measured static characteristics is compared with the two proposed electrothermal analysis methods. a) AM/AM, the data is normalized to the maximum value of measured gain, b) drain efficiency.

respectively. The pulsed signal power in the on state were $7 \mathrm{dBm}$ and $10 \mathrm{dBm}$, respectively. As it can be seen, the PA gain degrades with time for both input pulses, due to the heating. The PA gain degradations are about $0.65 \mathrm{~dB}$ and $0.4 \mathrm{~dB}$ for input pulses with $10 \mathrm{dBm}$ and $7 \mathrm{dBm}$ input powers, respectively.

The PA temperature was measured using one of the temperature sensing diode simultaneously with RF measurement. The relationship between the voltage of the sensing diode and its temperature was provided by the PA manufacturer under a fixed $10 \mu \mathrm{A}$ current bias. The measured diode voltage was prone to an electrical coupling from the input pulsed signal during measurement. Therefore, the measured voltage showed a DC offset synchronized with the input pulse on and off transitions. The corresponding offset was, therefore, removed from the diode's voltage to extract the correct voltage/temperature values. Fig. 14(b) shows the diode temperature, representing the PA temperature, versus time. As it can be observed, the temperature rises rapidly when the input signal is switched on and falls when the input pulse is turned off. Fig. 14 shows that the simulated gain and temperature agree well with measurement results.

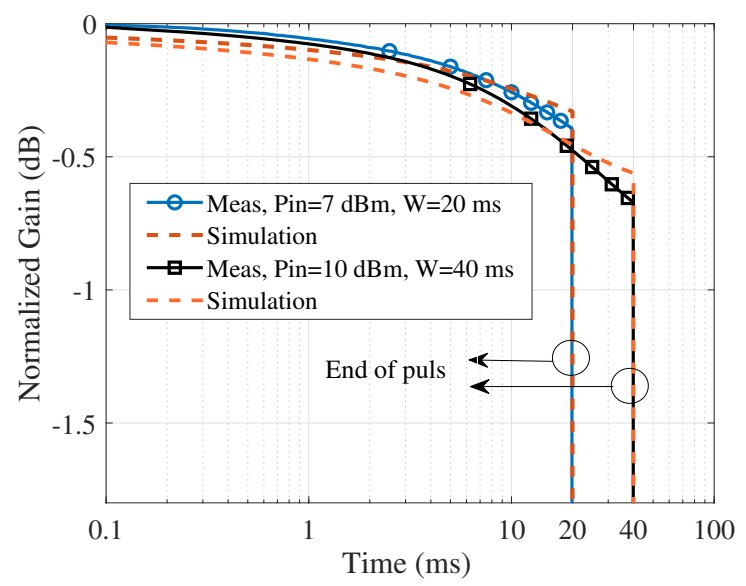

(a)

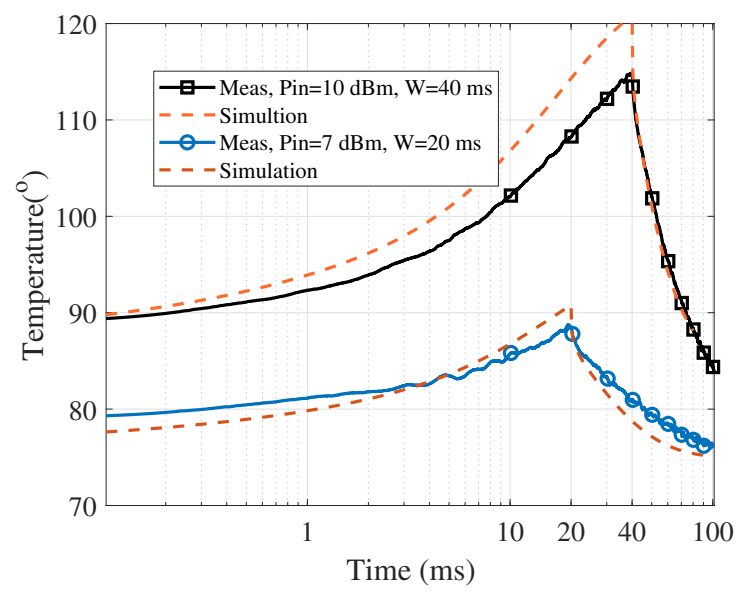

(b)

Fig. 14. Measured and simulated PA gain as well as temperature variation due to electrothermal effects. CW RF pulses with $20 \mathrm{~ms}, 40 \mathrm{~ms}$ pulse-widths (W) and pulse input power of 7 and $10 \mathrm{dBm}$ in the on state were applied to the PA DUT input. Duty cycle is $20 \%$ for both pulses. a) PA normalized gain, b) temperature variation. Simulation results were obtained from the model-based electrothermal analysis approach.

The measured results have been verified using the model-based electrothermal analysis method described in Section IV-C. Unfortunately, the circuit-based electrothermal analysis faced severe convergence issues in performing longtime envelope simulation. The reason can be the PA complex electrothermal netlist, including EM model as well as relatively long pulse width. In fact, even without any convergence issues, the use of circuit-based electrothermal approach leads to very long simulation time for temperature-aware envelope simulation. In these occasions, the use of model-based approach is essential to predict PA temperature-aware performance. In principle, the circuit-based approach can provide any type of thermally-aware circuit simulation. Therefore, it is most suitable at the PA design stage to optimize the schematic or layout from a joint thermal and electrical point of view.

\section{Modulated RF pulse}

For TDD communication systems, and in systems with burst-type communication patterns, the linearity performance 


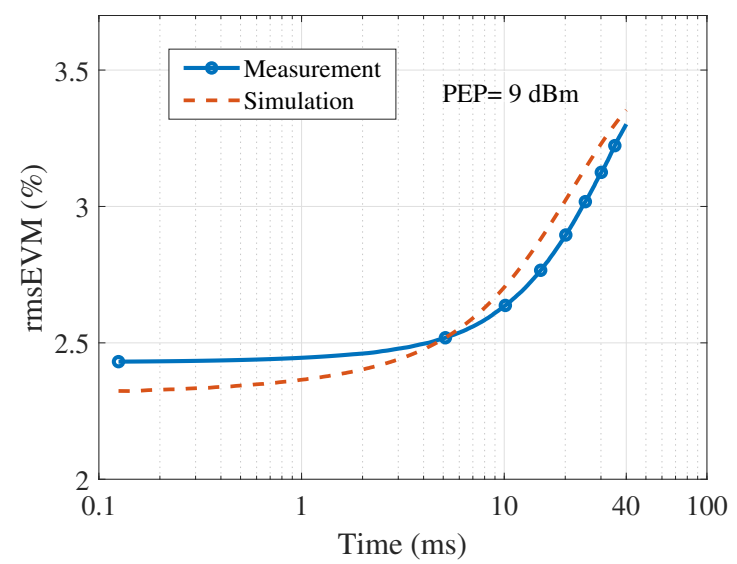

(a)

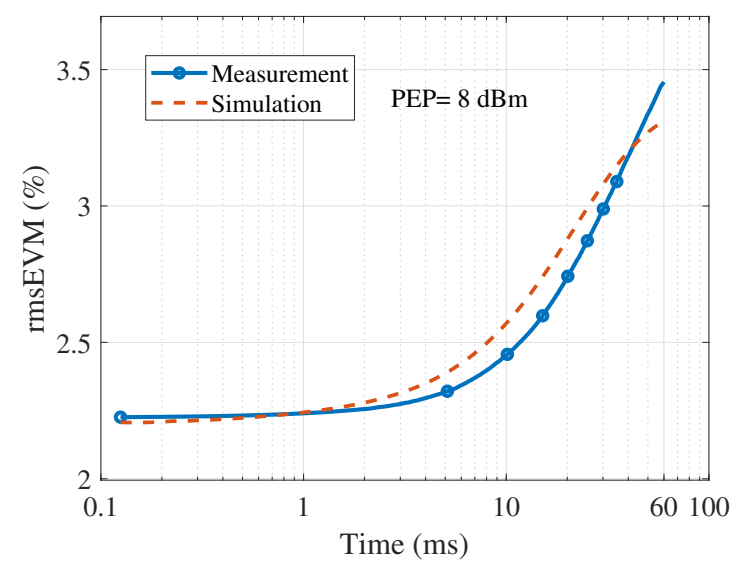

(b)

Fig. 15. Simulated and measured PA EVM (RMS value\%)versus time. Modulated signal pulses with a) $40 \mathrm{~ms}$ pulse-widths and Peak Envelope Power (PEP) of $9 \mathrm{dBm}$ and, b) $60 \mathrm{~ms}$ pulse-widths $8 \mathrm{dBm}$ PEP were injected to the PA input. Simulation results were obtained from model-based electrothermal analysis approach.

is required to be very high and stable versus time. An accurate characterization and modeling of EVM is critical for the evaluation and design of PAs under realistic pulsed modulated RF excitation due to thermal and transient PA behaviors. Pulsed modulated signal measurements have been performed to evaluate PA dynamic linearity characteristics. Therefore, 64 QAM modulated pulses with $6 \mathrm{MHz}$ bandwidth, $20 \%$ duty-cycle, and different pulse-widths were applied to the PA input.

The modulated signal measurements have been verified using the model-based electrothermal analysis method. Fig. 15 shows the RMS value of the measured EVM versus time for $40 \mathrm{~ms}$ and $60 \mathrm{~ms}$ pulse-widths and Peak Envelope Power (PEP) of $9 \mathrm{dBm}$ and $8 \mathrm{dBm}$, respectively. The EVM in Fig. 15 was calculated from a time frame of $0.1 \mathrm{~ms}$ and plotted versus time. This representation shows the effect of temperature variation on the PA dynamic linearity. As shown in Fig. 15(b), the EVM RMS value was degraded by approximately $1.3 \%$ and $0.7 \%$ for the input signals with $60 \mathrm{~ms}$ and $40 \mathrm{~ms}$ pulsewidths, respectively. Fig. 16 shows the constellation diagram

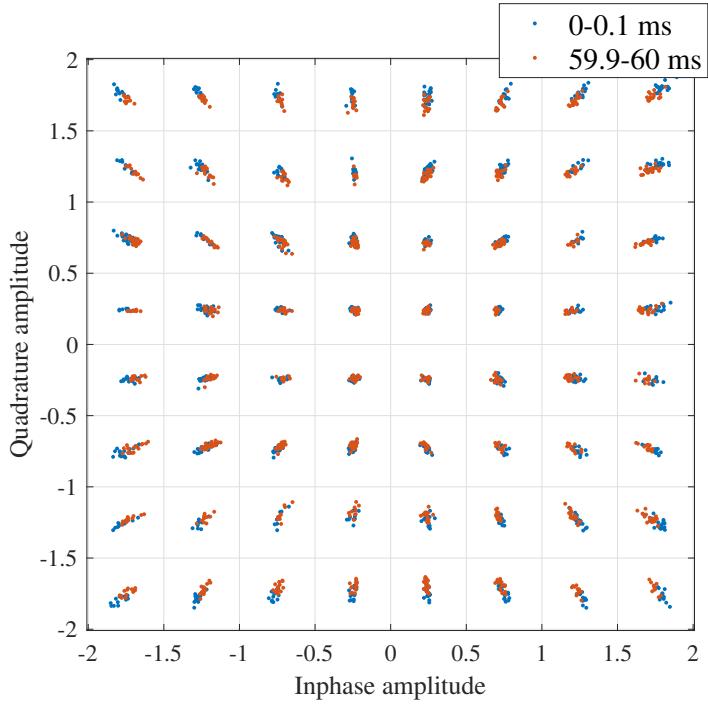

Fig. 16. Constellation diagram measured and plotted for two time frames, i.e. the first frame ( $0 \mathrm{~ms}<t<0.1 \mathrm{~ms}$, blue dots) and the last one (59.9 $\mathrm{ms}<t<60 \mathrm{~ms}$, red dots). The PA input is 64 QAM modulated pulse with $20 \%$ duty-cycle, $60 \mathrm{~ms}$ pulse-width and $8 \mathrm{dBm}$ PEP.

for the first and last time frames of the $60 \mathrm{~ms}$ pulse-width signal. It can be seen that the constellation related to the last time frame shrinks and linearity gets worse compared to the beginning of the pulse. Such behavior of linearity is often more challenging to compensate for using typical techniques such as DPD. Similar to the simulation of RF pulses in Section V-C, the steady-state were reached within less than 10 pulses and emulating the DC biasing condition. Fig. 15 shows good agreement between measurement and simulation results.

\section{CONCLUSIONS}

In this paper, first, an efficient methodology for PA thermal model extraction is proposed. The thermal model is extracted using 3-D thermal simulation data and a joint linear and nonlinear fitting algorithm. Based on the extracted thermal model, two efficient methodologies for electrothermal characterization of PA ICs are proposed.

First, a circuit-based electrothermal analysis is proposed which combines thermal- and electrical netlists into an efficient electro-thermal simulation. This method shows faster simulation speed compared to a commercial Electro-Thermal simulator as coupled 3-D thermal and electrical simulator. The second approach, called model-based electrothermal analysis, combines the PA thermal impedance matrix with PA nonlinear behavioral model to predict PA temperature-aware performance. The latter approach is suitable for system-level analyses in which electrothermal circuit simulation is too timeconsuming and complex to perform.

The proposed thermal model and both electrothermal analysis methods have been validated through static and pulsed RF signal measurements. The measured PA gain, temperature, and linearity results have shown a good agreement with electrothermal simulation results. Moreover, the dynamic PA performance, especially in terms of linearity, reveals the 
importance of electrothermal analysis for the correct prediction and design of PAs.

\section{ACKNOWLEDGEMENT}

The authors wish to thank NXP Semiconductors for providing the PA evaluation board. We also wish to acknowledge Keysight for the support with ADS Electro-Thermal and HeatWave thermal simulations.

\section{REFERENCES}

[1] S. Yoon, "Static and dynamic error vector magnitude behavior of 2.4 WIFI power amplifier," IEEE Trans. Microw. Theory Techn., vol. 55, no. 4, pp. 643-647, 2007.

[2] S. K. Yong and C.-C. Chong, "An overview of multigigabit wireless through millimeter wave technology: Potentials and technical challenges," EURASIP journal on wireless communications and networking, vol. 2007, no. 1, pp. 50-50, 2007.

[3] Y. Wang and R. Naylor, "Challenges in designing 5 WIFI 802.11ac WIFI power amplifiers," in 2014 IEEE Topical Conference on Power Amplifiers for Wireless and Radio Applications (PAWR), 2014, pp. 1618

[4] I. Ju, Y. Gong, and J. D. Cressler, "Highly linear high-power 802.11ac/ax WLAN SiGe HBT power amplifiers with a compact 2nd-harmonicshorted four-way transformer and a thermally compensating dynamic bias circuit," IEEE J. Solid-State Circuits, vol. 55, no. 9, pp. 2356-2370, 2020.

[5] D. Morgan, Z. Ma, J. Kim, M. Zierdt, and J. Pastalan, "A generalized memory polynomial model for digital predistortion of RF power amplifiers," IEEE Trans. Signal Process., vol. 54, no. 10, pp. 3852-3860, 2006.

[6] F. M. Ghannouchi and O. Hammi, "Behavioral modeling and predistortion," EEE Microw. Mag., vol. 10, no. 7, pp. 52-64, 2009.

[7] S. Boumaiza and F. Ghannouchi, "Thermal memory effects modeling and compensation in RF power amplifiers and predistortion linearizers," IEEE Trans. Microw. Theory Techn., vol. 51, no. 12, pp. 2427-2433, 2003.

[8] H. Najjari, C. Cordier, S. David, S. Bardy, and J.-B. Begueret, "Power amplifier with temperature adaptive biasing for improved DEVM," in 2018 IEEE Radio Frequency Integrated Circuits Symposium (RFIC), 2018, pp. 320-323.

[9] A. Samelis, E. Whittaker, M. Ball, A. Bruce, J. Nisbet, L. Lam and W. Vaillancourt, "Front-end modules with versatile dynamic EVM correction for 802.11 applications in the 2 WIFI band," in 2014 IEEE Topical Conference on Power Amplifiers for Wireless and Radio Applications (PAWR), 2014, pp. 10-12.

[10] V. d'Alessandro, M. de Magistris, A. Magnani, N. Rinaldi, and S. Russo, "Electrothermal macromodeling: an application to signal integrity analysis in highly integrated electronic systems," IEEE Trans. Compon. Packag. Manuf. Technol., vol. 3, no. 7, pp. 1237-1243, 2013.

[11] M. Pedram and S. Nazarian, "Thermal modeling, analysis, and management in VLSI circuits: Principles and methods," Proceedings of the IEEE, vol. 94, no. 8, pp. 1487-1501, 2006.

[12] M. T. Ozalas, "The impact of electro-thermal coupling on HBT power amplifiers," in 2014 IEEE Compound Semiconductor Integrated Circuit Symposium (CSICS), 2014, pp. 1-4.

[13] S. Wunsche, C. Clauss, P. Schwarz, and F. Winkler, "Electro-thermal circuit simulation using simulator coupling," IEEE Trans. Very Large Scale Integr. (VLSI) Syst., vol. 5, no. 3, pp. 277-282, 1997.

[14] W. van Petegem, B. Geeraerts, W. Sansen, and B. Graindourze, "Electrothermal simulation and design of integrated circuits," IEEE J. SolidState Circuits, vol. 29, no. 2, pp. 143-146, 1994.

[15] A. Chvála, D. Donoval, A. Šatka, M. Molnár, J. Marek, and P. Príbytný, "Advanced methodology for fast 3-D TCAD device/circuit electrothermal simulation and analysis of power HEMTs," IEEE Trans. Electron Devices, vol. 62, no. 3, pp. 828-834, 2015.

[16] A. Chvála, D. Donoval, J. Marek, P. Príbytný, M. Molnár, and M. Mikolášek, "Fast 3-D electrothermal device/circuit simulation of power superjunction MOSFET based on SDevice and HSPICE interaction," IEEE Trans. Electron Devices, vol. 61, no. 4, pp. 1116-1122, 2014.

[17] "ADS Electro-Thermal Simulator." [Online]. Available: https://www.keysight.com/se/en/product/W2349EP/pathwave-adselectro-thermal-simulator-element.html
[18] V. Košel, S. de Filippis, L. Chen, S. Decker, and A. Irace, "FEM simulation approach to investigate electro-thermal behavior of power transistors in 3-D," Microelectronics Reliability, vol. 53, no. 3, pp. 356-362, 2013. [Online]. Available: https://www.sciencedirect.com/science/article/pii/S0026271412004167

[19] L. Codecasa, "Thermal networks from heat wave equation," IEEE Trans Compon Packaging Manuf Technol., vol. 28, no. 1, pp. 14-22, 2005.

[20] — - "Compact models of dynamic thermal networks with many heat sources," IEEE Trans Compon Packaging Manuf Technol., vol. 30, no. 4 pp. 653-659, 2007.

[21] L. Codecasa, D. D'Amore, and P. Maffezzoni, "An Arnoldi based thermal network reduction method for electro-thermal analysis," IEEE Trans Compon Packaging Manuf Technol., vol. 26, no. 1, pp. 186-192, 2003.

[22] L. Codecasa, D. D'Amore, P. Maffezzoni, and W. Batty, "Analytical multipoint moment matching reduction of distributed thermal networks," IEEE Trans Compon Packaging Manuf Technol., vol. 27, no. 1, pp. 8795, 2004.

[23] L. Codecasa, D. D'Amore, and P. Maffezzoni, "Multipoint moment matching reduction from port responses of dynamic thermal networks," IEEE Trans Compon Packaging Manuf Technol., vol. 28, no. 4, pp. 605614,2005

[24] J. T. Hsu and L. Vu-Quoc, "A rational formulation of thermal circuit models for electrothermal simulation. I. finite element method [power electronic systems]," IEEE Trans. Circuits Syst. I. Fundam. Theory Appl., vol. 43, no. 9, pp. 721-732, 1996.

[25] - "A rational formulation of thermal circuit models for electrothermal simulation. II. model reduction techniques [power electronic systems]," IEEE Trans. Circuits Syst. I. Fundam. Theory Appl., vol. 43, no. 9, pp. 733-744, 1996.

[26] R. Sommet, D. Lopez, and R. Quéré, "From 3D thermal simulation of HBT devices to their thermal model integration into circuit simulators via Ritz vectors reduction technique," in ITherm 2002. Eighth Intersociety Conference on Thermal and Thermomechanical Phenomena in Electronic Systems (Cat. No. 02CH37258). IEEE, 2002, pp. 22-28.

[27] B. Shapiro and P. Mathai, "Interconnection of subsystem reduced-order models in the electro-thermal analysis of large systems," 2007.

[28] L. Codecasa, V. d'Alessandro, A. Magnani, and A. Irace, "Circuit-based electrothermal simulation of power devices by an ultrafast nonlinear MOR approach," IEEE Trans. Power Electron., vol. 31, no. 8, pp. 59065916, 2016.

[29] F. Ferranti, A. Magnani, V. d'Alessandro, S. Russo, N. Rinaldi, T. Dhaene, and M. de Magistris, "Effective electrothermal analysis of electronic devices and systems with parameterized macromodeling," IEEE Trans. Compon. Packag. Manuf. Technol., vol. 5, no. 6, pp. 788-796, 2015.

[30] L. Codecasa, D. D'Amore, and P. Maffezzoni, "Compact modeling of electrical devices for electrothermal analysis," IEEE Trans. Circuits Syst. I. Fundam. Theory Appl., vol. 50, no. 4, pp. 465-476, 2003.

[31] — - "Compact thermal networks for modeling packages," IEEE Trans Compon Packaging Manuf Technol., vol. 27, no. 1, pp. 96-103, 2004.

[32] M. Rencz, V. Szekely, and A. Poppe, "A methodology for the cosimulation of dynamic compact models of packages with the detailed models of boards," IEEE Trans Compon Packaging Manuf Technol., vol. 30, no. 3, pp. 367-374, 2007.

[33] C. Lasance, "Recent progress in compact thermal models," in Ninteenth Annual IEEE Semiconductor Thermal Measurement and Management Symposium, 2003., 2003, pp. 290-299.

[34] L. Codecasa, "Canonical forms of one-port passive distributed thermal networks," IEEE Trans Compon Packaging Manuf Technol., vol. 28, no. 1 , pp. 5-13, 2005.

[35] L. Codecasa, V. d'Alessandro, A. Magnani, N. Rinaldi, and P. J. Zampardi, "Fast novel thermal analysis simulation tool for integrated circuits (FANTASTIC)," in 20th International Workshop on Thermal Investigations of ICs and Systems, 2014, pp. 1-6.

[36] HSPICE® User Guide: Basic Simulation and Analysis, Synopsys.

[37] E. Baptista, K. Buisman, J. C. Vaz, and C. Fager, "Analysis of thermal coupling effects in integrated MIMO transmitters," in 2017 IEEE MTT-S International Microwave Symposium (IMS), 2017, pp. 75-78.

[38] K. Rasilainen, K. Buisman, K. Andersson, and C. Fager, "Multi-physical simulations and modelling of an integrated GaN-on-Si module concept for millimetre-wave communications," in 2020 IEEE 70th Electronic Components and Technology Conference (ECTC), 2020, pp. 1369-1375.

[39] C. Fager, K. Hausmair, T. Eriksson, and K. Buisman, "Analysis of thermal effects in active antenna array transmitters using a combined EM/circuit/thermal simulation technique," in 2015 Integrated Nonlinear Microwave and Millimetre-wave Circuits Workshop (INMMiC), 2015, pp. 1-3. 


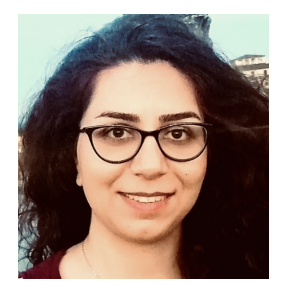

Parastoo Taghikhani received B.Sc and M.Sc degrees in electrical engineering, telecommunication from the Shahed University, Tehran, Iran in 2008 and 2011 respectively. She is currently pursuing a $\mathrm{PhD}$ degree at the Department of Microtechnology and Nanoscience (MC2), Chalmers University of Technology, Göteborg, Sweden. Her current research interests include modeling of active antenna arrays for mmWave MIMO transmitters. It also includes microwave and antenna design as well as multiphysics modeling of active antenna transmitters.

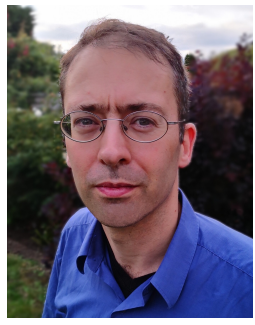

koen Buisman (Senior Member, IEEE) received the M.Sc. and Ph.D. degrees in microelectronics from the Delft University of Technology, Delft, The Netherlands, in 2004 and 2011, respectively. From 2004 to 2014, he was with the Delft Institute of Microsystems and Nanoelectronics, Delft University of Technology. In 2014, he joined the Chalmers University of Technology, Gothenburg, Sweden, where he is currently an Affiliated Associate Professor with the Microwave Electronics Laboratory, Department of Microtechnology and Nanoscience. In addition, in 2020, he joined the University of Surrey, Guildford, U.K., where he is currently a Reader in microwave and mm-wave electronics. He is also the Director of the Nonlinear Microwave Measurement and Modeling Laboratories, a joint University of Surrey/National Physical Laboratory, Teddington, U.K. He has authored or coauthored over 85 refereed journal and conference papers. He holds one patent. His current research interests include nonlinear device characterization, technology optimization, and design of linear transceivers for wireless systems.

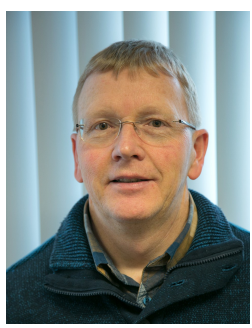

Martin Versleijen received his M.Sc degree in electronic engineering from the Technical University Eindhoven, Eindhoven, The Netherlands in 1985. He joined Philips Research Laboratories, Eindhoven, The Netherlands in 1987 where he worked on IC device modeling and characterization. In 1995 he joined Philips Semiconductors, since 2006 NXP Semiconductors, Nijmegen, The Netherlands, where he is involved in electronic design automation with a focus on tools and methodologies for RFIC and RF module design.

He has authored or coauthored more than 20 publications in international journals and conferences. His main research interests include wireless communications systems, with emphasis on high-efficiency wireless transmitters, tunable matching networks and RF/microwave topologies such as power amplifiers, rectifiers and DC/DC converters.

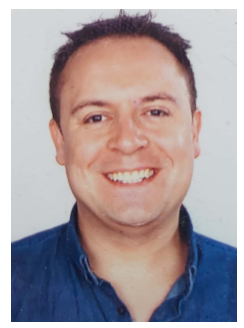

Jose-Ramon Perez-Cisneros received his Ph.D. degree (cum laude) from University of Zaragoza, Spain, in 2017. He currently holds a post-doc position at the Microwave Electronics Laboratory from Chalmers University of Technology, Sweden. From 2017 to 2019 he worked as researcher at the RF and Microwave Group from University of Cantabria, Spain. From 2013 to 2017 he was with the Institute of Engineering Research in Aragón (I3A) of the University of Zaragoza, being part of the Mobile Communications Group.

He has authored or coauthored more than 20 publications in international journals and conferences. His main research interests include wireless communications systems, with emphasis on high-efficiency wireless transmitters, tunable matching networks and $\mathrm{RF} /$ microwave topologies such as power amplifiers, rectifiers and DC/DC converters.

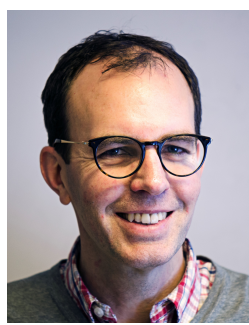

Christian Fager Christian Fager received his Ph.D. degree from Chalmers University of Technology, Sweden, in 2003. He became Full Professor at the same university in 2019 and is currently Head of the Microwave Electronics Laboratory. He has authored or co-authored more than 200 publications in international journals and conferences, where he has made significant contributions to the area of linear and energy efficient power amplifiers and transmitters for wireless communications.

Dr. Fager serves as Associate Editor of IEEE Microwave Magazine and IEEE Microwave and Wireless Components Letters. $\mathrm{He}$ is representative for Sweden, Norway and Iceland in the European Microwave Association (EuMA) and member of the IEEE MTT-S Technical Committee on Wireless Communications. He served as chair of the 2021 IEEE Topical Conference on RF/microwave Power Amplifiers and TPC cochair for the 2020 European Microwave Integrated Circuits Conference. Dr. Fager received Chalmers Supervisor of the Year Award in 2018 and the IEEE International Microwave Symposium Best Student Paper Award in 2002. 